\title{
APLICACIONES DE CÁÑAMO COMO ALTERNATIVA RENTABLE A LA REACTIVACIÓN ECONÓMICA DE ECUADOR TRAS LA PANDEMIA DE COVID-19.
}

\section{Hemp applications as a profitable alternative to the economic reactivation of Ecuador after the Covid-19 pandemic.}

Henry Adrian Gallegos Dávila* iD

Universidad Central del Ecuador, Facultad de Ingeniería Química, Quito, Ecuador.

*hagallegos@uce.edu.ec

$\mathrm{R}$ esumen

El presente estudio muestra un análisis del aprovechamiento del cáñamo como una potencial alternativa para la reactivación económica del Ecuador tras la crisis que atraviesa durante y después de la pandemia de Covid-19. Para ello se estudiaron 3 puntos principales que son: el enfoque económico, donde se muestra la situación actual de la economía ecuatoriana y el flujo de dinero que genera el cáñamo a nivel mundial es decir los principales importadores y exportadores; el enfoque legal, donde se exponen las modificaciones en leyes ecuatorianas respecto al cáñamo y algunos beneficios que estas conllevan; y finalmente el enfoque Agrícola e industrial donde se evidencia la potencialidad productiva del cáñamo y productos derivados del cáñamo. Finalmente, de manera general se puede concluir que el aprovechamiento del cáñamo apoyado de las vigentes reformas de COIP, permitirá crear numerosas plazas de trabajo en diferentes sectores como el agrícola, comercial e industrial. Paralelamente se proyecta incrementar el nivel de exportaciones y fomentar el comercio interno; es decir la compra y venta de materia prima o productos derivados del cáñamo.

Palabras claves: Cáñamo Importaciones, Cáñamo Exportaciones, Productos Cáñamo, Covid 19.

\section{A bstract}

This study shows an analysis of the use of hemp as a potential alternative for the economic revival of Ecuador after the crisis experienced during and after the Covid-19 pandemic. To this end, three main points were studied: the economic approach, which shows the current situation of the Ecuadorian economy and the luxury of money generated by hemp worldwide, i.e. the main importers and exporters; the legal approach, which sets out the changes in Ecuadorian laws with respect to hemp and some of the benefits that these entail and finally, the agricultural and industrial approach where the productive potential of hemp and hemp-derived products is highlighted. It can be generally concluded that hemp utilization is supported by the current COIP reforms will allow the creation of numerous jobs in different sectors such as agriculture, commercial and industrial. At the same time, it is planned to increase the level of exports and promote domestic trade; is the purchase and sale of raw materials or products derived from hemp.

Keywords: Hemp Imports, Hemp Exports, Hemp products, Covid 19. 


\section{INTRODUCCIÓN}

La industrialización del cáñamo, es decir la elaboración masiva de productos derivados del cáñamo como toda nueva industria generará numerosas plazas de trabajo lo cual favorece la dinamización económica en diferentes áreas productivas del Ecuador. Empezando por la reactivación y renovación de la industria agrícola (producción de materias primas), pasando por la industria de los procesos y finalmente la comercialización del producto e incluso como materia prima (importación y exportación de cáñamo).

En la actualidad, estamos en deuda con nuestro país en el ámbito industrial y esta puede ser la oportunidad de reivindicarnos. Una vez obtenida la materia prima y apoyados por las vigentes reformas del Código Orgánico Integral Penal en cuanto al uso autorizado del cáñamo con fines de producción, industrialización y comercialización. Se abre un amplio abanico de opciones en cuanto a procesos y productos que involucran el cáñamo como se ha realizado en diferentes países a lo largo de la historia, obteniendo así productos como fibras textiles, plásticos, papel, aceites y actualmente incluso casas a base de cáñamo.

Es importante tomar en cuenta que esta es una vía factible hacia la reactivación económica ya que como se menciona empieza desde la siembra y cosecha del cáñamo dinamizando los sectores agrícolas para continuar con una revolución industrial donde podemos obtener gran diversidad de productos.

\section{MATERIALES Y MÉTODOS}

La metodología empleada en el presente trabajo fue cualitativa y cuantitativa, llevada a cabo mediante investigación bibliográfica. Para lo cual se recopiló y analizó información bibliográfica enfocada al estudio del cáñamo en Ecuador y a nivel mundial de diferentes autores como: publicaciones de artículos científicos, tesis, capítulos de libros, revistas y bases de datos. También se tomaron como referencias diferentes videoconferencias relacionadas con el cáñamo y su aprovechamiento en Ecuador.

La revisión de los documentos se realizó tan- to en inglés como español, publicados desde el año 2010 hasta el 2020. Empleando el buscador de literatura académica Google Scholar y otras bases de datos como repositorios de las universidades, PubMed, Redalyc, Elsevier, ScienceDirect o SciELO; las palabras usadas se concentraron en título y palabras clave, relacionadas a estudios realizados sobre el cáñamo.

Con el objetivo de presentar un análisis del aprovechamiento del cáñamo como una potencial alternativa para la reactivación económica, se filtró la información más relevante y actual, aplicando criterios de selección como el periodo de estudio, área geográfica, accesibilidad, autenticidad, relevancia en nuestro tema de estudio, idioma, y tipo de documento. Finalmente, mediante una lectura crítica y detallada, se eligieron los documentos para el correspondiente estado del arte; categorizando la información en tres grupos (enfoque económico, enfoque legal y enfoque agrícola e industrial).

Además, los datos recopilados se procesaron mediante herramientas informáticas (Excel) para una mejor visualización.

\section{RESULTADOS Y DISCUSIÓN}

\section{Enfoque económico (una breve mirada del pro- blema):}

La situación económica que atraviesa Ecuador durante y tras la pandemia muestra una gran caída. Como se puede evidenciar en las previsiones macroeconómicas del (1), donde se plantearon tres escenarios con diferentes supuestos como la producción de petróleo, las importaciones y exportaciones totales, inversión pública y privada, los gastos del gobierno y las remesas. De lo cual se estimó que el PIB, principal indicador macroeconómico a nivel global, decaería entre 7.27 y 9.59 puntos para 2020. Esto, de manera general se traduce en una fuerte crisis económica y un notorio aumento en las tasas de desempleo; lo que es evidente debido a la falta de productividad de las empresas y negocios en general, durante los primeros meses de la pandemia.

Por otro lado, el precio del petróleo disminuyó 
respecto al 2019, cuando se cotizó en 51,3 dólares por barril, mientras que, en marzo de este año se valorizó en 23 dólares por barril. (1). Además, como consecuencia de la erosión del cauce del Río Coca en las provincias de Napo y Sucumbíos, la economía ecuatoriana se vio afectada debido a la rotura de las tuberías del $(\mathrm{SOTE})^{1}$ y del $(\mathrm{OCP})^{2}$ en abril del presente año.

\section{El Camaño en números:}

Es evidente el beneficio que se ha obtenido a nivel global de esta planta ancestral y además es un reflejo de su rentabilidad, ya que, a diferencia de otros productos, donde sus importaciones y exportaciones tienden a decaer en el tiempo. Según International Trade Statistics, el número de Importaciones y Exportaciones Globales expresada en dólares americanos ha aumentado en función del tiempo (Gráfico 1). Principalmente el nivel de exportaciones donde para el año 2019 a nivel global se registran 22'892 000 [dólares] a diferencia de su año más bajo (2014) con 2'966 000 [dólares] (2).

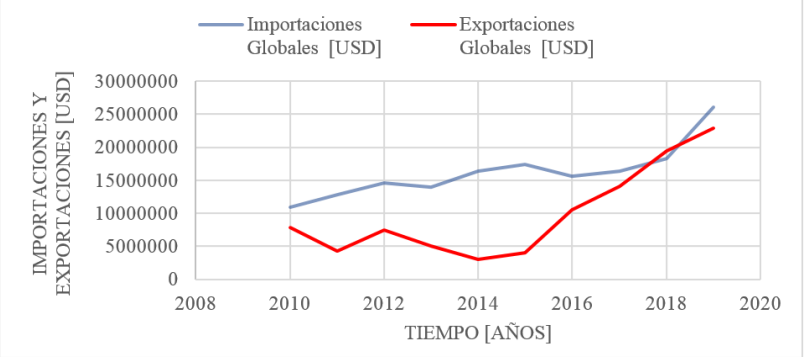

Grafico 1. Importaciones y Exportaciones globales de Cáñamo medido en dólares en función del tiempo.

Elaborado a partir de: (2).

Alemania lidera el mercado de importaciones con valores crecientes del producto "5302 Cáñamo (Cannabis sativa L., (en bruto o trabajado, pero sin hilar; estopas y desperdicios de cáñamo, incluido los desperdicios de hilados y las hilachas)"; así para 2010 importaron, 1167 [miles de dólares americanos], para el año 2015 se obtuvieron 4784 [miles de dólares]. Para el año 2019, se registran 3646 [miles de dólares], seguido de España con 3206 [miles de dólares], y Republica Checa 3144 [miles de dólares] (2). De esto se puede concluir que Alemania puede abrir un amplio mercado del producto a diversos países que se están uniendo a la iniciativa de sacarle provecho al Cáñamo Industrial, desarrollando un potencial mercado primario como se encuentra en otros estudios "Plan de Negocios y potencialidad del cultivo de cáñamo en Ecuador" (3).

Por otro lado, Países Bajos, Suiza y Croacia ha sacado un gran provecho siendo los principales exportadores generando un creciente flujo de efectivo en torno a las exportaciones de esta planta en la última década (Gráfico 2), donde para el año 2019 se registran valores de 6292, 5650 y 2515 [miles de dólares] respectivamente (2).

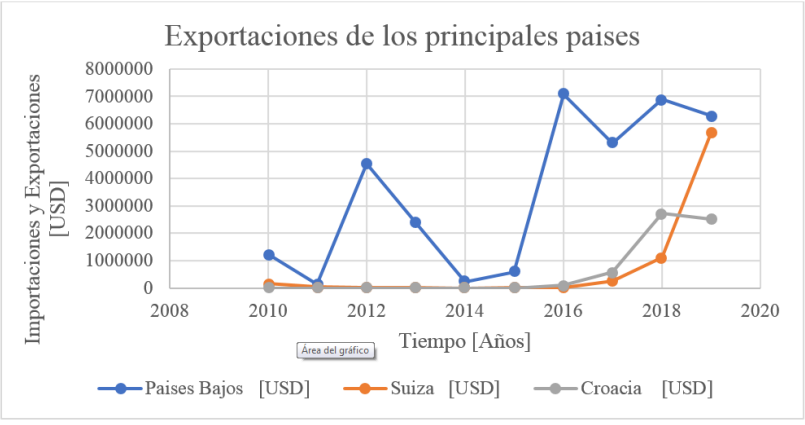

Gráfico 2. Exportaciones de los principales países exportadores de Cáñamo, expresadas en dólares en función del tiempo.

Elaborado a partir de: (2)

Finalmente (4), se plantea un análisis comparativo donde; la producción de cannabis no psicoactivo rico CBD, a cielo abierto implica niveles de costes de producción, riesgo bajos y rentabilidad altos. Por otro lado, la siembra en invernadero presenta costos de producción riesgo y rentabilidad muy altos qué se debe tomar en cuenta a la hora de incursionar en este negocio.

\section{El Enfoque legal.}

Grandes cambios se aproximan en Ecuador, gracias a la actual situación jurídica del cáñamo. Donde una serie de modificaciones en los artículos han abierto las puertas al aprovechamiento de esta planta, ya sea con fines comerciales (producción e importación de materia prima y derivados de cáñamo) o industriales, impulsando y desarrollando la obtención de productos derivados del cáñamo industrial.

En el webinar abierto, organizado por el Ministerio de Agricultura y Ganadería el miércoles 20 de mayo del 2020 en el cual se expone la normativa y oportunidades del cultivo de cannabis en Ecuador; Andrés Luque, subsecretario de producción agrícola del MAG, menciona con respecto a la reforma en el código orgánico integral penal 
(COIP); que el 24 de diciembre del 2019 la asamblea decide por mayoría de votos la despenalización del cultivo y producción del cannabis en Ecuador con un contenido inferior al 1\% de THC (componente psicoactivo del cannabis. Además, se instaura al $\mathrm{MAG}^{3}$ como órgano competente para emitir la regulación respecto a la reforma que entró en vigor el 21 de junio del 2020. Esta reformulación en el COIP ${ }^{4}$ posibilita dos tipos de cosecha: el cáñamo para uso industrial y el cannabis no psicoactivo o cáñamo; el cual debido a que presenta propiedades fenotípicas similares al cannabis psicoactivo, no permitirá el auto cultivo. Por otra parte, al tener un plazo de 120 días, las disposiciones transitorias tienen como fecha límite el 19 de octubre del 2020 para expedir la regulación a partir de la ley vigente (4).

Algunas ventajas de la normativa del Ecuador respecto a otras normativas del cáñamo se mencionan que en Ecuador el MAG, será el encargado desde la importación y la producción de semillas hasta la exportación de biomasa o aceite crudo lo cual agilitará los procesos ya que, en otros países las licencias son emitidas por varias instituciones. Ecuador también permitirá la comercialización de biomasa para evitar errores que han cometido otros países, que enfocados en mejorar la producción industrial interna se estancaron, no pudieron procesar su materia prima y tampoco exportarla. Además, las licencias serán intransferible y sus costos serán variables, con determinado costo por hectárea (4).

El Ministerio de Agricultura y ganadería menciona que las licencias solo podrán ser solicitadas por personas jurídicas, entidades públicas, cooperativas o asociaciones de pequeños y medianos productores residentes en el país; los cuales deberán presentar la respectiva documentación sobre las inversiones y estados financieros que demuestren la legalidad de los recursos que se emplearán en dicho proyecto.

Otro requisito principal, es tener una promesa de compra de su producto ya sea del mercado interno o externo; en otras palabras, deberán tener un comprador (destino final) asegurado. Además, se deberán presentar antecedentes penales para el trámite, con el fin de evitar actos de corrupción por parte de personas que quieran sacar provecho de la nueva normativa (5).

Así, Ecuador se suma a los países de Latinoamérica que se adelantaron a la iniciativa; como Uruguay, que el 7 de enero del 2014 autoriza el uso del cannabis para fines científicos y médicos, industrialización para uso farmacéutico (Ley 27.350). Chile que el 7 de diciembre del 2015, Permite el uso terapéutico del cannabis durante un corto periodo de tiempo, regulaciones especiales establecidas para otorgar licencias, para control y supervisión para el cultivo industrial y comercial, así como para la investigación científica o la elaboración de fitofármacos que contengan THC (Ley 20.000 y Decreto 84). Colombia en 2016, legalizó el acceso al cannabis medicinal, permitiendo el cultivo, procesamiento y exportación comercial de productos de marihuana medicinal para uso sanitario y científico. Argentina el 19 de abril de 2017, permite investigación médica y científica (Ley. 19.172 y Decreto 46-015) (6).

\section{El enfoque Agrícola e Industrial.}

Luque (4), menciona que el aprovechamiento del cáñamo se puede enfocar en la obtención de materia prima, lo cual fomenta el desarrollo agrícola ya que el proceso abarca desde la compraventa de semillas, pasando por la siembra, cultivo y terminando con la cosecha del cáñamo para su posterior comercialización (interna o externa). En este punto, se plantea un análisis comparativo entre las principales zonas productivas del Ecuador y se concluye que las zonas costa y la sierra son las más adecuadas para la producción del cáñamo; ya que el oriente debido a su alto porcentaje de humedad, la cosecha es muy propensa a la formación de hongos. Además, se encuentra que la sierra presenta ciclos de producción más largo y cosechas con contenido de CBD más alto, a diferencia de la región costa, cuyos ciclos de producción son más cortos y sus cosechas tienen contenido de CBD más bajo.

Por otro lado, también se aprovecha estas materias primas para el desarrollo industrial interno. Desde este punto de vista, se puede obtener una diversidad muy amplia de productos elaborados a partir del cáñamo que van desde: bolsas bio- 
degradables elaboradas con celulosa de cáñamo dirigida a grandes y medianas empresas (7), pasando por la fabricación de papel de cáñamo (8), aceites de semilla de cáñamo con aplicaciones gastronómicas o la extracción de aceites ricos en CBD destinados a la industria cosmética o farmacéutica (9), para tratamientos de epilepsia, esclerosis múltiple, enfermedades inflamatorias crónicas como la poliartritis crónica, enfermedades de inflamación intestinal, entre otros (10). Algunas propiedades terapéuticas de los principales cannabinoides que se pueden aprovechar en este punto son: el Cannabidiol (CBD) como ansiolítico, antipsicótico, analgésico y antiespasmódico; Delta-9- tetrahidrocannabinol $(\Delta 9-T H C)$ como analgésico, antiemético y antiinflamatorio; Cannabinol ( $\mathrm{CBN})$ como sedante, anticonvulsivo, antibiótico y antiinflamatorio y finalmente el Cannabicromeo (CBC) como antiinflamatorio, antimicótico y analgésico (11).

Otros productos que se pueden obtener son biocombustibles como bioetanol a partir de biomasa de cáñamo (12), bebidas alcohólicas aromatizadas con flores de cáñamo (13). También se puede producir materiales estructurales novedosos compuestos de cáñamo industrial y $(\mathrm{HDPE})^{5}$ reciclado con muy buenas propiedades mecánicas (14), incluso se pueden elaborar ladrillos a base de cáñamo industrial (15), para su posterior aplicación en la construcción de casas ecológicas (16).

Por otra parte, estudios muestran como resultado de un análisis comparativo entre Uruguay, Chile y Argentina (países que nos llevan ventaja en la despenalización y aprovechamiento esta planta) que los 3 países coinciden en contemplar la vía de la importación, pero, además, dos de ellos consideran que es necesario ir más allá y regular otras formas de producción de derivados de la planta garantizando un acceso a los mismos. Es decir, plantean promover la producción masiva de derivados del cáñamo y no solamente materia prima para exportación; enfocándose además de las exportaciones, en el consumo interno de estos productos (17).

Así, por ejemplo, entre los primeros registros de aprovechamiento del cáñamo en la industria ecuatoriana. Laboratorios Beautik, firma que opera en Durán introducirá en el mercado una línea de champú, acondicionador, crema para peinar y mascarilla capilar que se elabora con aceite de la semilla de cannabis rico en ácidos grasos esenciales y omega 3, 6 y 9 (18).

\section{Manejo y cultivo del cáñamo industrial}

Hay que tomar en cuenta que las propiedades del producto obtenido van a depender de muchos factores cómo una variedad de la semilla, lugar dónde se va a cultivar es decir su calidad de suelo y condiciones climáticas, por ello la importancia del manejo del cultivo en función del uso qué se quiera dar el producto final. En este caso nos enfocamos en el cáñamo industrial con un contenido ( $\mathrm{THC}<1 \%$ ) desde su siembra, su cultivo y manejo; pasando por la obtención de materias primas cómo son semillas, cañamones ricos en aceite y diferentes fibras que pueden ser comercializados como materia prima o aprovecharlas en algún proceso industrial para la obtención de productos textiles, aceites, cosméticos, materiales compuestos, medicamentos, entre otros (19) .

Entre las principales necesidades del cultivo está la tierra, ya que el cáñamo necesita tierras sueltas (aproximadamente 15\% de arena fina, 15\% de humus y pequeñas cantidades de arcilla para evitar encharcamientos) ricas en nutrientes (principalmente calcio y potasio). Además, se debe controlar la salinidad y que el $\mathrm{pH}$ no sea menor de 5.5 (evitar elevada acidez). La cantidad de luz durante el crecimiento es de al menos 8 horas diarias de sol y 7 horas de oscuridad ininterrumpidas. Finalmente, la cantidad de agua y riego varía según el clima y temperatura de la zona; pero de manera general se requiere una precipitación pluvial mínima de aproximadamente $700 \mathrm{~mm}$ al año y humedad abundante (20).

En cuanto al clima, la temperatura ambiental ideal en el día oscila entre los 20 y $25^{\circ} \mathrm{C}$ y por la noche entre 13 y $17^{\circ} \mathrm{C}$ en un entorno templado con adecuada humedad ya que son susceptibles a las bajas temperaturas (pueden modificar sus propiedades). Otro factor importante son los abonos empleados ya que se estima, que el cáñamo requiere $15 \mathrm{~kg}$ de fertilizante por $\mathrm{kg}$ de fi- 
bra obtenido; ya que esta compone el $6.5 \%$ de la masa vegetal. Otros estudios han medido la cantidad de $\left(\mathrm{N}, \mathrm{P}_{2} \mathrm{O}_{5}, \mathrm{~K}_{2} \mathrm{O}\right)$ que el cáñamo extrae del suelo durante un ciclo de vida, obteniendo (102; 66 y 117) [kg/ha] respectivamente. De ser necesaria la aplicación de abonos; se recomienda que sean orgánicos (estiércol animal, humus orgánico y otros) y abonar mínimo dos meses antes de la siembra evitando el uso de turba ya que esta acidifica el suelo $(21,22)$.

\section{Calculadora del crecimiento y rendimiento}

En base a estudios compilatorios de campo de Seedcx.com y la Universidad de Ridgetown / Keneex Ltd. Se ha desarrollado una herramienta de cálculo del cáñamo industrial en función del número de hectáreas sembradas; donde, en base a aproximaciones se puede obtener los requisitos de cultivo, la población y densidad de siembra, la cantidad de nutrientes (fosfato, nitrógeno y potasa) en kilogramo requeridos, el rendimiento los valores monetarios potenciales de su venta al por mayor (New Age Hemp).

Así, por ejemplo, tomando una base de cálculo, para 10 hectáreas de cultivo de cáñamo se requieren $1000 \mathrm{~kg}$ de nitrógeno, $700 \mathrm{~kg}$ de potasa $\mathrm{y}$ $450 \mathrm{~kg}$ de fosfato. Los rendimientos de fibra estimados son $447780 \mathrm{Kg}$ de fibra BAST (con un valor monetario de 4447800 €) y $1383760 \mathrm{~kg}$ de fibra HURD (con un valor monetario de 7223 227. $2 €$ ) y para semillas de aceite son 177047.15 litros de aceite de semilla (valor monetario de 2 $478660.1 €$ ) y $938.98 \mathrm{~kg}$ de extracto de CBD con un valor monetario de $23474500 €$ (New Age Hemp).

Altos rendimientos de fibra se pueden conseguir con densidades de siembra entre 250 a 400 semillas viables por metro cuadrado (50-60 libras por acre); sin embargo, dependerá del tipo de suelo, la fertilidad de este de tipo de cultivo. Por otro lado, la producción de semilla o grano requerirá densidades más bajas entre 35 y 45 libras por acre (New Age Hemp).

\section{Clasificación sistémica:}

El Cannabis se encuentra dentro del grupo de las
Angiospermas, en la clase Magnoliopsidas, subclase Hamamélidas, orden Urticales, en la familia Cannabaceas donde encontramos dos especies, el lúpulo (Humulus lupulos L.), y el cáñamo (Cannabis sativa L.) (23).

Dentro del Cannabis sativa, se encuentran tres subespecies (indica, rudelaris y sativa) distinguidas por su origen, tipo de semilla y el manejo de su crecimiento. Así, Cannabis sativa Indica típica del sudeste asiático son relativamente pequeñas, pero bastante ramificadas, de forma cónica pueden alcanzar 1.5 metros de altura; por otro lado, Cannabis sativa Rudelaris originaria de $\mathrm{Ru}-$ sia normalmente de menor ramificación, pero de mayor altura, alcanza entre dos y cinco metros de altura y finalmente la Cannabis sativa Sativa originaria de Europa y África presenta altura y ramificación moderada, alcanzando entre 1.5 y 2 metros de alto (24).

En la actualidad, gracias al desarrollo agrónomo y tecnológico se han obtenido una gran variedad especies modificas y por ello es necesaria una clasificación más práctica. De un lado, esta las plantas con porcentajes bajos de THC (0.2-0.3) $\%$ con fines principalmente industriales como el cáñamo industrial y el cannabis no psicoactivo (25) y por otro lado variedades como (Skunk, o Silver Haze) con mayor contenido de THC (5-10) $\%$ para fines terapéuticos e investigativos (20).

La planta del Cannabis germina, crece, se desarrolla al pasar por ocho etapas o estadios vegetativos así: al germinar, la raíz sale de la extremidad afilada de la semilla; luego de $48 \mathrm{~h}$ de la germinación, la raíz tiene una longitud aproximada de 2.5 a $4 \mathrm{~cm}$ (a partir de aquí, el crecimiento se ralentiza); transcurridas $72-96$ horas comienza a desarrollarse bajo tierra un sistema de raicillas laterales, los cotiledones (aún pegados), empiezan a asomar a la superficie y a ejercer presión para deshacerse de la envoltura de la semilla; al llegar al día cinco el tallo de la plántula comienza a levantarse, (se cae la envoltura y se abren los cotiledones); el tallo crece constantemente hasta el día diez aproximadamente (aparece el primero par de hojas verdaderas); después el segundo y tercer par de hojas; al comienzo de la semana tres, se caen los cotiledones (la planta crecerá 
bien si dispone de suficiente luz). Después en los meses tres y cuatro los machos echarán flores, y más tarde las hembras y finalmente se produce la fecundación, donde los machos primero se vuelven amarillos y luego mueren; mientras que las hembras, producen cañamones y después empiezan a marchitarse (20).

\section{Ventajas del cultivo de cáñamo}

Entre las principales ventajas del cultivo de cáñamo esta su asombrosa velocidad de crecimiento; ya que, este puede alcanzar alturas de aproximadamente cuatro metros entre tres y cuatro meses (dependiendo de las condiciones del cultivo); además, en las primeras dos a tres semanas se puede evidenciar a nivel general del cultivo, el desarrollo de una especie de tapiz verde y espeso que posteriormente se convertirá en un frondoso bosque de cáñamo erradicado de malas hierbas del suelo. Otra ventaja es que puede ser cultivado orgánicamente evitando el empleo de químicos nocivos para el medioambiente, es decir que no requiere de pesticidas y herbicidas (20).

Sin embargo, para mejorar su rendimiento, se prefieren suelos ricos en nutrientes (de baja acidez y bajo contenido de metales pesados) y un adecuado suministro de agua. A la par, mejora el rendimiento de futuros cultivos del suelo debido a que devuelve al terreno aproximadamente $38-40 \%$ de la extracción de minerales que realiza durante su desarrollo.

También puede ser manejado como un cultivo de rotación, alternando cosechas de cáñamo con otros productos como por ejemplo maíz, papas, trigo, remolacha, entre otros. Y se dice que un campo de trigo o de papas es $10 \%$ más productivo si el año anterior ha sido plantado con cáñamo ya que, casi tan rápido como su crecimiento hacia arriba crece también su fina red de raíces que se extienden por la tierra esponjándola y aireándola, preparando el suelo para próximos cultivos (26).

Estos aspectos lo convierten en un cultivo económicamente viable, debido a su rápido crecimiento y sus bajos requerimientos de mantenimiento. El cáñamo además, supera a otros productos como el trigo, la soya, heno, entre otros en rendimientos netos (dólares por área de cultivo); ya sea en el precio bala de la fibra/rendimiento que se estima en 500 dólares por hectárea y precio alto de la fibra/rendimiento 1250 dólares por hectárea (26).

\section{CONCLUSIONES}

Ecuador, evidentemente puede enfocar su reactivación económica en el aprovechamiento del cáñamo ya que debido a las reformas del COIP puestas en vigencia desde el 21 de junio del 2020, se permite el cultivo y producción del cannabis con un contenido inferior al $1 \%$ de THC (componente psicoactivo de la planta).

Esta reactivación económica se puede enfocar en la producción de cáñamo como materia prima para su posterior comercialización (interna o externa) o en el aprovechamiento de estas materias primas para el desarrollo industrial interno. Así, la reactivación empieza desde la compraventa de semillas y la producción de materia prima, es decir, arranca en el sector agrícola, generando numerosas plazas de trabajo desde el campo, ya sea en la siembra, producción y cosecha de cáñamo hasta la posterior comercialización interna y exportación de materia prima hacia los principales mercados importadores de cáñamo como Alemania, España, República Checa e incluso Estados Unidos.

Por otro lado, a nivel interno se puede promover el desarrollo del sector industrial con el aprovechamiento de esta planta que tiene una amplia gama de aplicaciones en diferentes sectores industriales.

Así, por ejemplo, en la industria alimenticia, farmacéutica, la industrial de los cosméticos y la industria en general; ya que, como se mencionó en este estudio de la planta se pueden obtener desde aceites, plásticos, papel, biocombustibles hasta la elaboración de casas.

En resumen, se puede generar una gran cantidad de plazas de trabajo, se incrementa el nivel de exportaciones, se estimula el comercio interno ya sea por la compra o venta de materia prima o productos derivados del cáñamo. 
1. Banco Central del Ecuador. El covid-19 pasa factura a la economía ecuatoriana: decrecerá entre 7,3\% y 9,6\% en 2020 [Internet]. 2020 [cited 2020 Jul 19]. Available from: https://www.bce.fin.ec/ index.php/boletines-de-prensa-archivo/item/1366-el-covid-19-pasa-factura-a-la-economia-ecuatoriana-decrecera-entre-73-y-96-en-2020

2. International Trade Statistics. Trade Map - Lista de los importadores para el producto seleccionado (Cáñamo (Cannabis sativa L.), en bruto o trabajado, pero sin hilar; estopas y desperdicios de ...) [Internet]. 2020 [cited $2020 \mathrm{Jul}$ 20]. Available from: https://www.trademap.org/tradestat/ Country_SelProduct_TS.aspx?nvpm =3\%7C\%7C\%7C\%7C\%7C5302\%7C\%7C\%7C4\%7C1\%7C1\%7C1\%7C2\%7C1\%7C2\%7C1\%7C1\%7C1

3. Pino Herrera O. ESTUDIO DE PRE FACTIBILIDAD PARA LA CREACIÓN DE UNA EMPRESA PRODUCTORA Y PROCESADORA DE FIBRA DE CÁÑAMO INDUSTRIAL EN LA PROVINCIA DE PICHINCHA PARA LA EXPORTACIÓN AL MERCADO ALEMÁN EN EL PERIODO 2019-2029 [Internet]. 2019 [cited 2020 Jul 19]. p. 148. Available from: http://repositorio.puce.edu.ec/bitstream/handle/22000/15967/ESTUDIO DE PRE FACTIBILIDAD PARA LA CREACIÓN DE UNA EMPRESA PRODUCTORA Y PROCESADORA DE FIBRA DE CANAMO INDUSTRIAL EN LA $~ 1$.pdf? sequence=1\&isAllowed $=\mathrm{y}$

4. Luque A. Normativa y Oportunidades del Cultivo de Cannabis en Ecuador - YouTube [Internet]. Youtube ; 2020 [cited 2020 Aug 24]. Available from: https://www.youtube.com/watch?v=TLudjg1jwWU\&t=3179s

5. Ministerio de Agricultura Y Ganadería. MAG explica cómo será el uso industrial del cáñamo Ministerio de Agricultura y Ganadería [Internet]. 2020 [cited 2020 Jul 22]. Available from: https:// www.agricultura.gob.ec/mag-explica-como-sera-el-uso-industrial-del-canamo/

6. Aguilar S, Gutiérrez V, Sánchez L, Nougier M. Políticas y prácticas sobre cannabis medicinal en el mundo [Internet]. Ciudad de México ; 2018 Apr [cited 2020 Aug 19]. Available from: http:// fileserver.idpc.net/library/Medicinal cannabis briefing_SPA_FINAL.PDF

7. Cayllahua B, Mendoza Suárez J;, Alberto C, Ortega R, Jessica G, Salas S, et al. Biohemp Perú Item Type info:eu-repo/semantics/bachelorThesis [Internet]. Umiversidad Peruana de Ciencias Aplicadas (UPC); 2020 [cited 2020 Jul 15]. Available from: http://hdl.handle.net/10757/651992

8. Arencibia F, Peña B, Goyeneche J. La fabricación del papel de cáñamo: eco alternativa sostenible en zonas de alta vulnerabilidad. Mundo Fesc [Internet]. 2020 Jan;10(19):67-79. Available from: https://www.fesc.edu.co/Revistas/OJS/index.php/mundofesc/article/view/511/552

9. Fuentes E, Acurio L. El Cañamo (Cannabis sativa L.) para uso industrial y farmacéutico: una visión desde la industria alimentaria. CienciAmérica. 2020 Dec 16;9(4):99.

10. Schluttenhofer C, Yuan L. Challenges towards Revitalizing Hemp: A Multifaceted Crop. 2017 Nov [cited 2021 May 16];22(11):917-29. Available from: https:/www.sciencedirect.com/science/ article/pii/S1360138517301772

11. Daya. Cannabis medicinal: una breve guía sobre usos y efectos - Fundación Daya [Internet]. Fundación Daya. 2016 [cited 2020 Aug 19]. Available from: http://www.fundaciondaya.org/cannbis-medicnal-usos-efectos-tipos-de-cannabis/

12. Zhao J, Xu Y, Wang W, Griffin J, Roozeboom K, Wang D. Bioconversion of industrial hemp biomass for bioethanol production: A review. Fuel [Internet]. 2020 Dec 1 [cited 2021 May 14];281:118725. Available from: https://www.sciencedirect.com/science/article/pii/S001623612031721X

13. Ascrizzi R, Iannone M, Cinque G, Marianelli A, Pistelli L, Flamini G. "Hemping" the drinks: Aromatizing alcoholic beverages with a blend of Cannabis sativa L. flowers. Food Chem [Internet]. 2020 Sep 30 [cited 2021 May 15];325:126909. Available from: https://www.sciencedirect.com/ science/article/pii/S0308814620307718

14. Lu N, Johnson R. HEMP FIBER REINFORCED COMPOSITE WITH RECYCLED HIGH DENSITY POLYETHYLENE AND PRODUCTION THEREOF [Internet]. US 2017/0121473 
A1, 2017 [cited 2020 Jul 22]. Available from: https://patentimages.storage.googleapis.com/1a/ 3b/00/4a75cf157fb546/US20170121473A1.pdf

15. Ospina Pedraza Oscar Andres. Diseño de modelo de negocio verde a partir de la producción de ladrillos a base cáñamo industrial [Internet]. reponame: Repositorio Institucional Universidad El Bosque. [Bogotá]: Universidad El Bosque; 2019 [cited 2020 Jul 21]. Available from: http://repositorio.unbosque.edu.co/handle/20.500.12495/2617

16. Shea A, Lawrence M, Walker P. Hygrothermal performance of an experimental hemp-lime building. Constr Build Mater. 2012 Nov 1;36:270-5.

17. Mora Bassante JE. "LA PRODUCCIÓN DE CANNABIS CON FINES TERAPÉUTICOS PARA TUTELAR EL DERECHO AL ACCESO A LA SALUD A TRAVÉS DE ENTIDADES AUTÓNOMAS, COMUNITARIAS Y DE MEDICINA ALTERNATIVA." [Internet]. [Ambato]: Pontificia Universidad Católica del Ecuador ; 2018 [cited 2020 Aug 19]. Available from: https://repositorio. pucesa.edu.ec/bitstream/123456789/2504/1/76787.pdf

18. Reyes J. La industria cosmética ecuatoriana comienza uso de cannabis como materia prima | Economía | Noticias | El Universo. El Universo [Internet]. 2020 Jul 17 [cited 2020 Aug 12]; Available from: https://www.eluniverso.com/noticias/2020/07/17/nota/7908537/cannabis-ecuador-industria-capilar-medicinal

19. Company G, Cano J, Zaragoza Á. El legado del cáñamo en Callosa de Segura (Alicante): clave en su industrialización y la configuración de su territorio. Geographos [Internet]. 2020 Jan 2 [cited 2021 May 17];11(122):1-25. Available from: https://rua.ua.es/dspace/bitstream/10045/103368/1/ German_Company.pdf

20. Acosta X. Agroecología del cáñamo. La Fertilidad de la Tierra [Internet]. 2001 [cited 2020 Sep 2];29-32. Available from: https://www.mapa.gob.es/ministerio/pags/biblioteca/revistas/pdf_Ferti/ Ferti_2001_6_29_32.pdf

21. Fassio A, Rodríguez M, Ceretta S. CáñAmo (Cannabis sativa L.) [Internet]. Montevideo; 2013 [cited 2020 May 17]. Available from: http://www.inia.org.uy

22. New Age Hemp. Calculadora del crecimiento, Rendimiento y salida | [Internet]. New Age Hemp. [cited 2020 Sep 2]. Available from: https://newagehemp.es/calculadora-del-crecimiento-rendimiento-y-salida/

23. Integrated Taxonomic Information System (ITIS). Cannabis sativa L [Internet]. 2014 [cited 2021 May 16]. Available from: https://www.itis.gov/servlet/SingleRpt/SingleRpt?search_topic=TSN\&search_value=19109\#null

24. García J. Ensayo De Variedades De Cáñamo En La Vega Baja del Segura [Internet]. [Elche]: Universidad Miguel Hernández; 2016. Available from: http://dspace.umh.es/bitstream/11000/2971/1/ TFG García Hernández\%2C Jorge.pdf

25. Callaway J. A more reliable evaluation of hemp THC levels is necessary and possible. J Ind Hemp [Internet]. 2008 Oct 13 [cited 2020 May 17];13(2):117-44. Available from: https://www.tandfonline.com/doi/abs/10.1080/15377880802391142

26. Gamarra J. TRABAJO FINAL DE CAÑAMO INDUSTRIAL [Internet]. Academia. 2020 [cited 2020 Sep 2]. Available from: https://www.academia.edu/3289598/TRABAJO_FINAL_DE_CANAMO_INDUSTRIAL 